Supplementary Materials for

\title{
Photooxidation of Isoprene by Titanium Oxide Cluster Anions with Dimensions up to a Nanosize
}

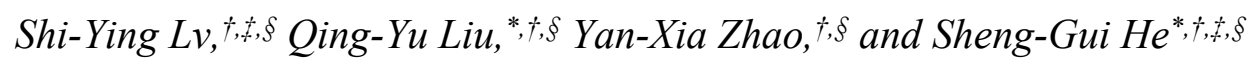

†State Key Laboratory for Structural Chemistry of Unstable and Stable Species, Institute of Chemistry, Chinese Academy of Sciences, Beijing 100190, P. R. China

tUniversity of Chinese Academy of Sciences, Beijing 100049, P. R. China

${ }^{\S}$ Beijing National Laboratory for Molecular Sciences and CAS Research/Education Center of Excellence in Molecular Sciences, Beijing 100190, P. R. China 
(a1)
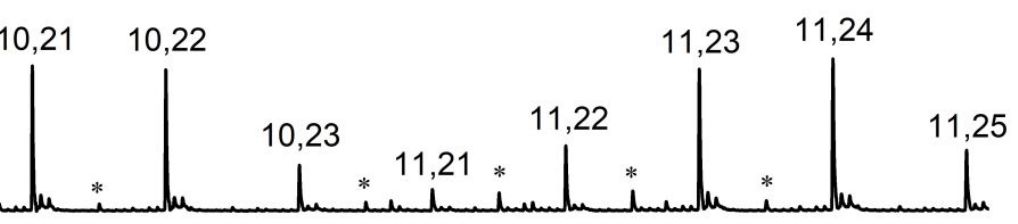

(a2)

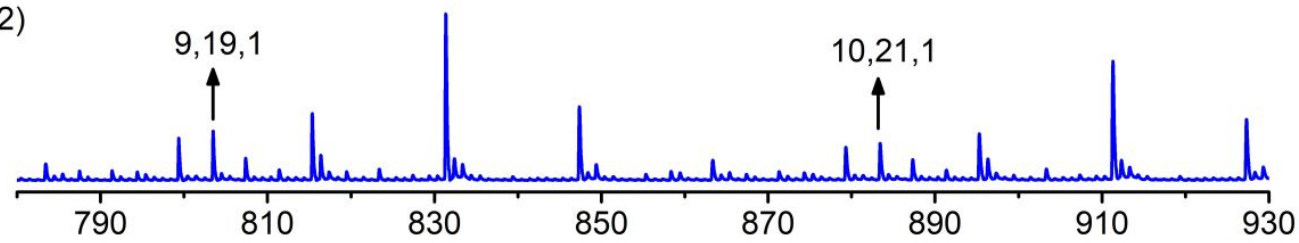

(b1)

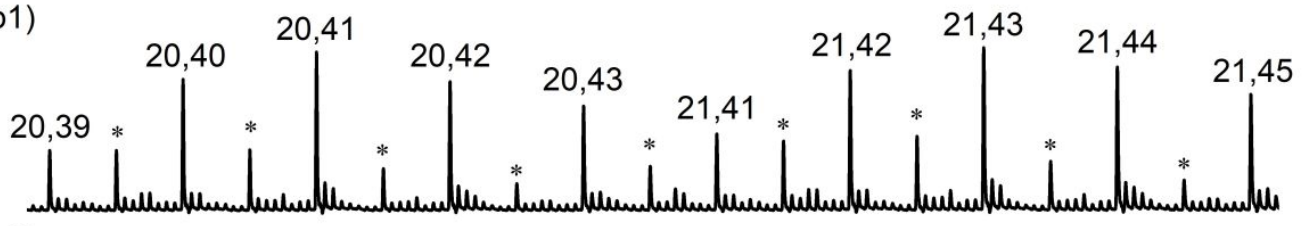

(b2)

$$
19,39,1
$$

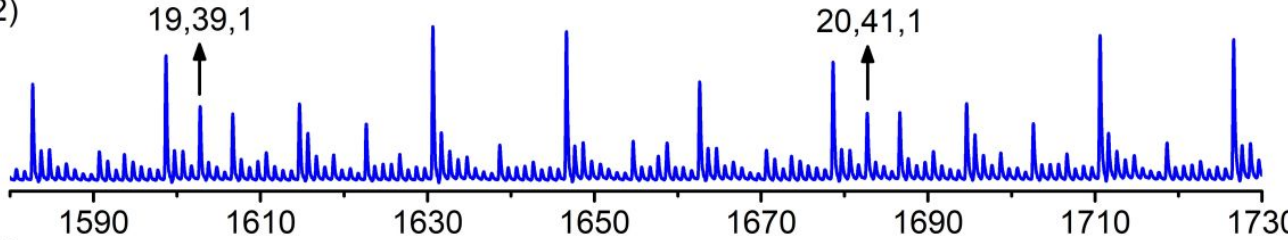

(c1)

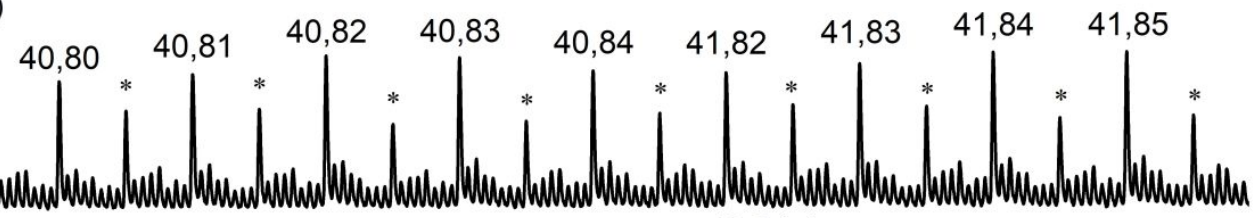

(c2) $39,79,1 \quad 39,80,1 \quad 39,81,1 \quad 40,80,1 \quad 40,81,1 \quad 40,83,1$

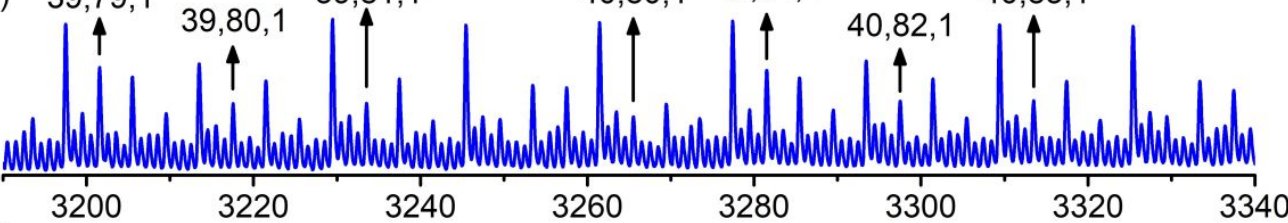

(d1)

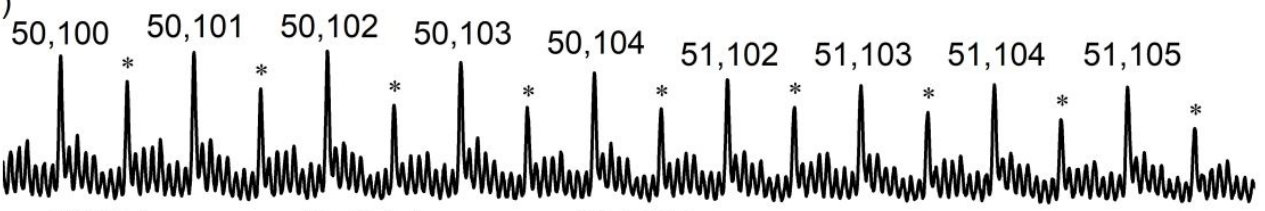

(d2)

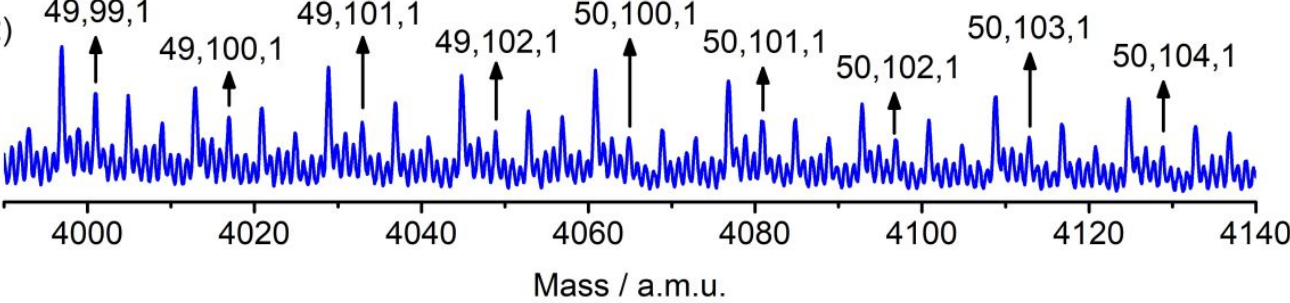

Figure S1. Selected TOF mass spectra for the reactions of $\mathrm{Ti}_{x} \mathrm{O}_{y}{ }^{-}(\mathrm{a} 1, \mathrm{~b} 1, \mathrm{c} 1$, and $\mathrm{d} 1)$ with $0.1 \mathrm{~Pa} \mathrm{C}_{5} \mathrm{H}_{8}(\mathrm{a} 2, \mathrm{~b} 2, \mathrm{c} 2$, and $\mathrm{d} 2)$ in the fast flow reactor. The association products $\mathrm{Ti}_{x} \mathrm{O}_{y}\left(\mathrm{C}_{5} \mathrm{H}_{8}\right)^{-}$are labeled as " $x, y, 1$ ". The peaks marked with asterisks originate from the Ca impurities in the sample of ${ }^{48} \mathrm{Ti}$ powder. 


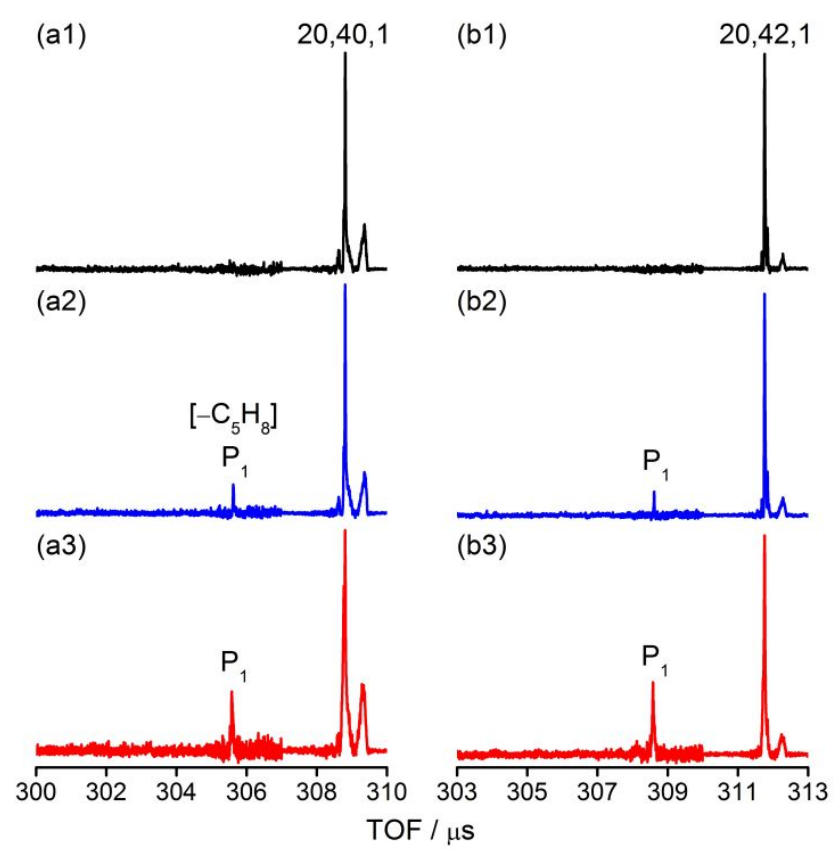

Figure S2. Mass spectra of $\mathrm{Ti}_{20} \mathrm{O}_{40} \mathrm{C}_{5} \mathrm{H}_{8}{ }^{-}(\Delta=-1)$ and $\mathrm{Ti}_{20} \mathrm{O}_{42} \mathrm{C}_{5} \mathrm{H}_{8}{ }^{-}(\Delta=3)$ for mass selections (a1, b1, and c1), photoreactions at $355 \mathrm{~nm}(\mathrm{a} 2, \mathrm{~b} 2$, and $\mathrm{c} 2)$, and CID reactions with $\mathrm{He}(\mathrm{a} 3, \mathrm{~b} 3$, and $\mathrm{c} 3)$. The relative intensities around the product peaks amplified by a factor of 25 . The laser fluence is $29.3 \pm 0.4 \mathrm{~mJ} / \mathrm{cm}^{2}$. 


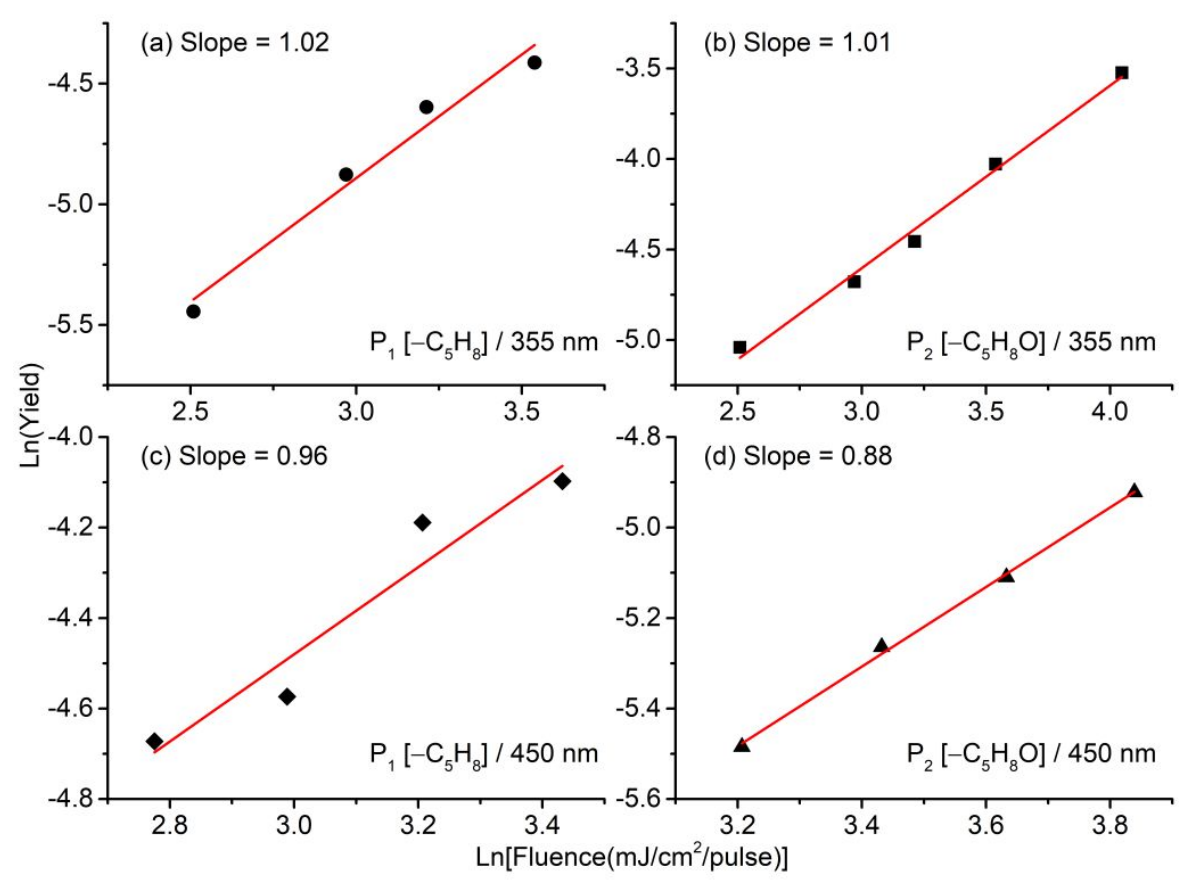

Figure S3. The Ln-Ln plots for product yield of $\mathrm{P}_{1}$ and $\mathrm{P}_{2}$ versus the laser fluence on $\left(\mathrm{TiO}_{2}\right)_{15} \mathrm{OC}_{5} \mathrm{H}_{8}{ }^{-}$at $355 \mathrm{~nm}$ (a and b) and $450 \mathrm{~nm}$ (c and d), respectively. 


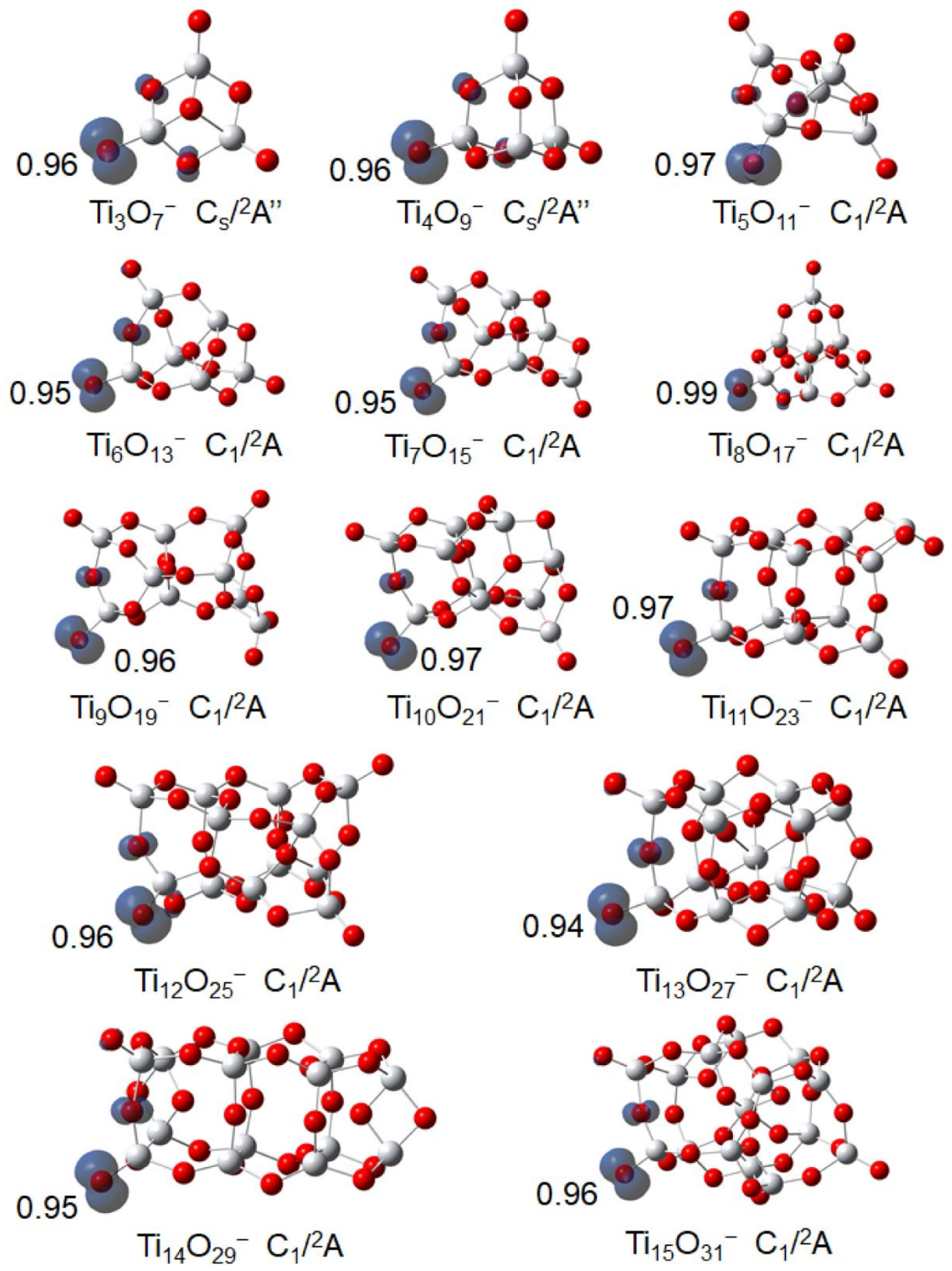

Figure S4. DFT calculated structures and unpaired spin density distributions for the lowest-lying isomers of $\left(\mathrm{TiO}_{2}\right)_{x} \mathrm{O}^{-}(3 \leq x \leq 15)$. The unpaired spin densities $\left(\right.$ in $\left.\mu_{\mathrm{B}}\right)$ on the oxygen atoms are shown. The structures of $\left(\mathrm{TiO}_{2}\right)_{x} \mathrm{O}^{-}(3 \leq x \leq 8)$ have been reported. ${ }^{1}$ 

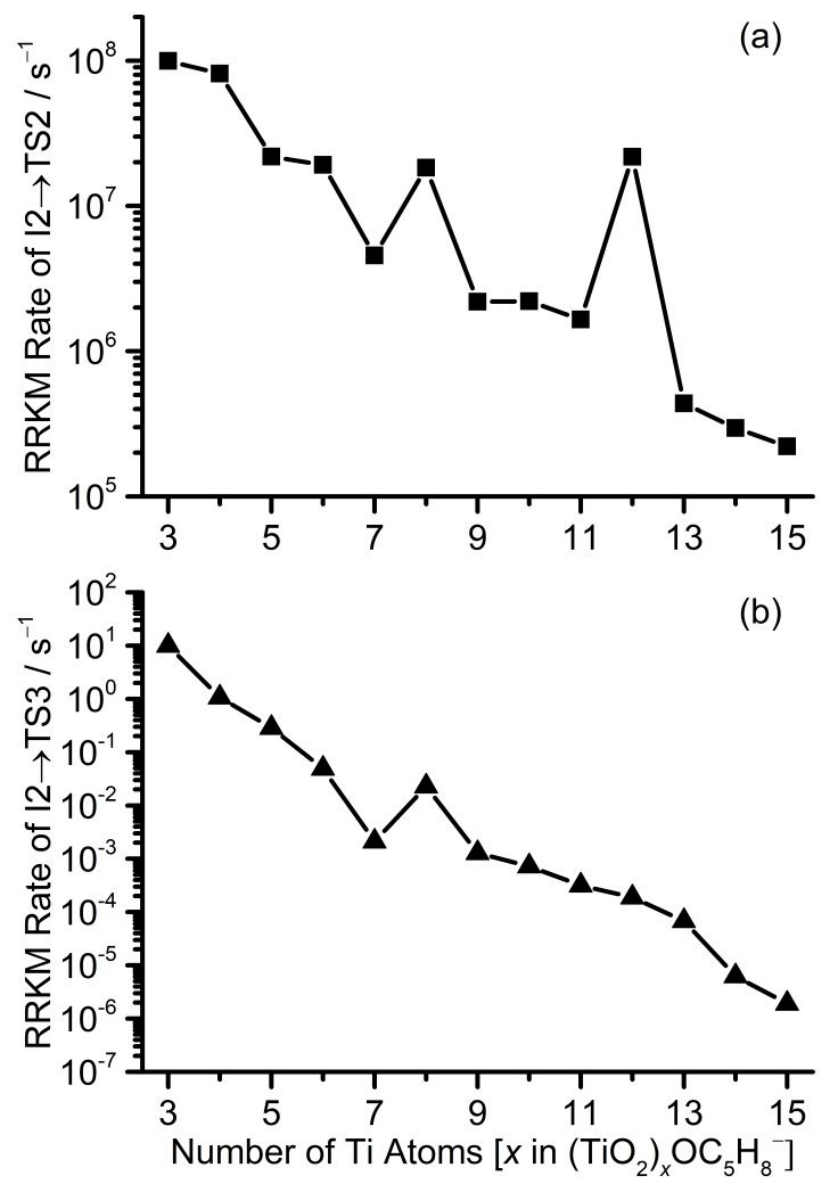

Figure S5. The RRKM calculated rates of $\mathrm{I} 2 \rightarrow \mathrm{TS} 2 \rightarrow \mathrm{I} 3(\mathrm{a})$ and $\mathrm{I} 2 \rightarrow \mathrm{TS} 3 \rightarrow \mathrm{I} 4$ (b). The rates of collisions between the clusters and the He buffer gas in the fast flow reactor is about $10^{7} \mathrm{~s}^{-1}$, which is much larger than the rates (panel b) for transformation of I2 or I3 into I4 through TS3. As a result, the experimentally generated $\left(\mathrm{TiO}_{2}\right)_{x} \mathrm{OC}_{5} \mathrm{H}_{8}{ }^{-}$ could be I 2 or I3. As I 2 is slightly more stable than I3 (Table S1), the I2 is considered as the primiary species in this work. 


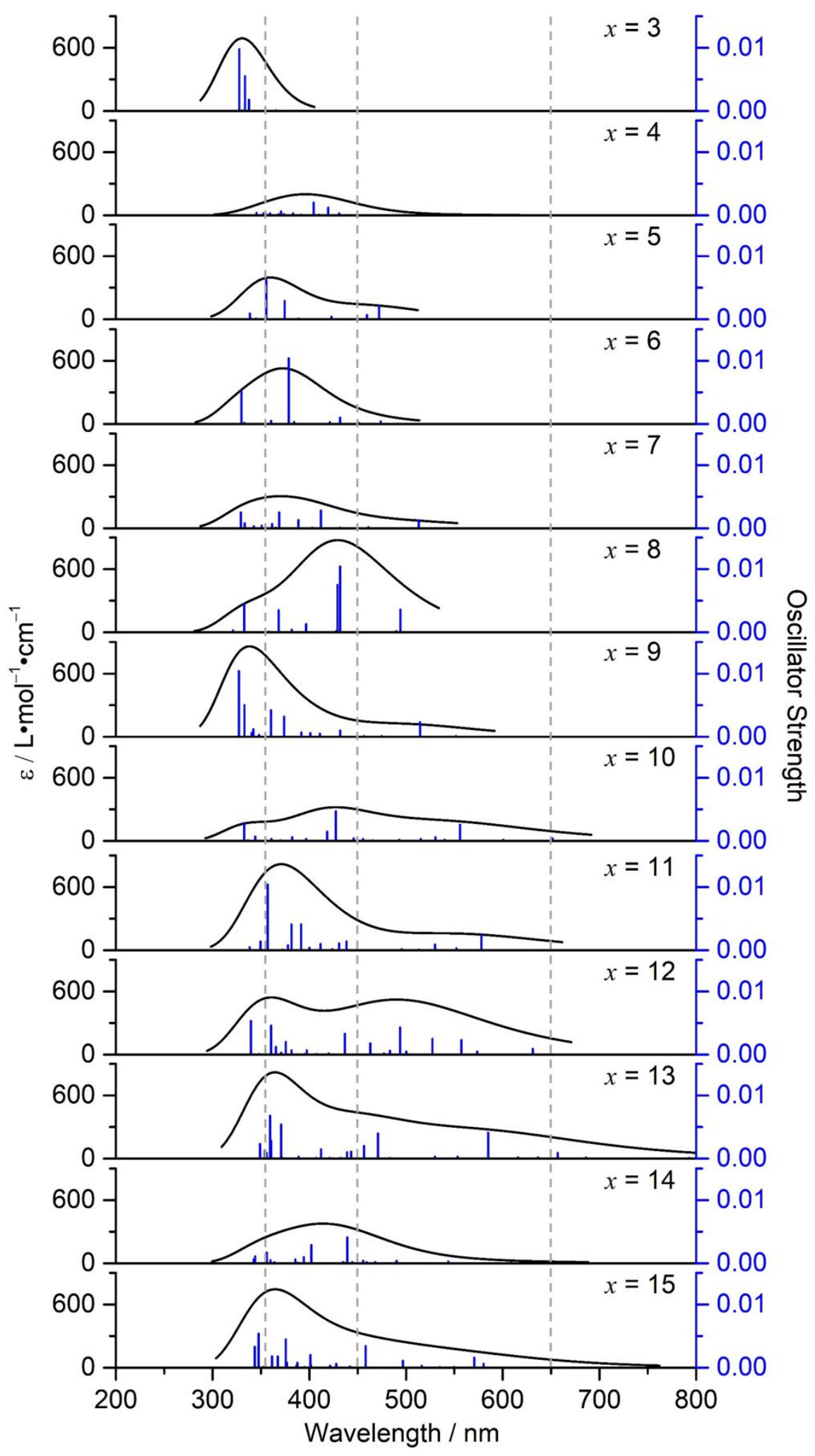

Figure S6. The DFT calculated UV-Vis absorption spectra of $\left(\mathrm{TiO}_{2}\right)_{x} \mathrm{OC}_{5} \mathrm{H}_{8}{ }^{-}(x=3-$ 15). The wavelength at $355 \mathrm{~nm}, 450 \mathrm{~nm}$, and $650 \mathrm{~nm}$ are marked with dotted lines. The bar plot is broadened by using Gaussian function with FWHM of $0.6667 \mathrm{eV}$. The area under the curve that broadened from per unit oscillator strength is 28700 empirically. ${ }^{2}$ 
Table S1. DFT calculated relative zero-point vibration corrected energies $\left(\Delta H_{0}\right.$ in eV) of I2, TS2, I3, TS3, I4, I5 and products $\left[\left(\mathrm{TiO}_{2}\right)_{x}{ }^{-}+\mathrm{C}_{5} \mathrm{H}_{8} \mathrm{O}\right]$ with respect to the corresponding separated reactants $\left[\left(\mathrm{TiO}_{2}\right)_{x} \mathrm{O}^{-}+\mathrm{C}_{5} \mathrm{H}_{8}\right](x=3-15)$. The stability of the highly symmetric structure of $\left(\mathrm{TiO}_{2}\right)_{13}{ }^{-}$may lead to the relatively large exothermicity of $\left(\mathrm{TiO}_{2}\right)_{13} \mathrm{O}^{-}+\mathrm{C}_{5} \mathrm{H}_{8} \rightarrow\left(\mathrm{TiO}_{2}\right)_{13}{ }^{-}+\mathrm{C}_{5} \mathrm{H}_{8} \mathrm{O}(\Delta H=-0.52 \mathrm{eV})$.

\begin{tabular}{cccccccc}
\hline$x$ & I2 & TS2 & I3 & TS3 & I4 & I5 & Products \\
\hline 3 & -1.57 & -0.89 & -1.57 & -0.18 & -1.96 & -1.94 & 0.53 \\
4 & -1.53 & -0.86 & -1.52 & -0.14 & -1.90 & -1.88 & 0.22 \\
5 & -1.66 & -1.01 & -1.65 & -0.24 & -2.00 & -1.98 & 0.18 \\
7 & -1.62 & -0.95 & -1.60 & -0.19 & -1.95 & -1.94 & 0.05 \\
8 & -1.83 & -1.17 & -1.80 & -0.40 & -2.14 & -2.13 & -0.01 \\
9 & -1.73 & -1.07 & -1.70 & -0.30 & -2.03 & -2.03 & 0.03 \\
10 & -1.68 & -1.02 & -1.66 & -0.25 & -2.01 & -2.00 & -0.05 \\
11 & -1.77 & -1.11 & -1.73 & -0.36 & -2.08 & -2.08 & -0.10 \\
12 & -1.78 & -1.12 & -1.75 & -0.34 & -2.10 & -2.11 & -0.14 \\
13 & -1.68 & -1.03 & -1.65 & -0.24 & -2.02 & -2.04 & -0.52 \\
14 & -1.66 & -0.99 & -1.63 & -0.22 & -1.97 & -1.97 & -0.06 \\
15 & -1.70 & -1.04 & -1.69 & -0.29 & -2.03 & -2.03 & -0.20 \\
\hline
\end{tabular}


Table S2. The net negative charges $\left(\Delta Q\right.$, in unit of e) transferrd from $\mathrm{C}_{5} \mathrm{H}_{8}$ to $\left(\mathrm{TiO}_{2}\right)_{x} \mathrm{O}^{-}$ $(3 \leq x \leq 15)$ cluster in $\mathrm{I} 2\left[\left(\mathrm{TiO}_{2}\right)_{x} \mathrm{OC}_{5} \mathrm{H}_{8}{ }^{-}, x=3-15\right]$.

\begin{tabular}{cccccccc}
\hline$x$ & 3 & 4 & 5 & 6 & 7 & 8 & 9 \\
\hline$\Delta Q$ & -0.2329 & -0.2282 & -0.2821 & -0.2921 & -0.2849 & -0.3125 & -0.3051 \\
\hline$x$ & 10 & 11 & 12 & 13 & 14 & 15 & \\
\hline$\Delta Q$ & -0.3048 & -0.3220 & -0.3571 & -0.3319 & -0.3114 & -0.3278 & \\
\hline
\end{tabular}


Table S3. Three orbital pairs which have largest contributions to the excitation of each $\left(\mathrm{TiO}_{2}\right)_{x} \mathrm{OC}_{5} \mathrm{H}_{8}{ }^{-}(3 \leq x \leq 15)$ cluster. The excited states with the excitation energy closest to $355 \mathrm{~nm}(3.492 \mathrm{eV})$ and $450 \mathrm{~nm}(2.755 \mathrm{eV})$ were chosen.

\begin{tabular}{|c|c|c|c|c|c|c|}
\hline Cluster & $\begin{array}{c}\text { Excitation } \\
\text { Energy }(\mathrm{eV})\end{array}$ & Orbital Pairs & $\begin{array}{c}\text { Absolute } \\
\text { Contribution(\%) }\end{array}$ & $\begin{array}{c}\text { Excitation } \\
\text { Energy }(\mathrm{eV})\end{array}$ & Orbital Pairs & $\begin{array}{c}\text { Absolute } \\
\text { Contribution(\%) }\end{array}$ \\
\hline \multirow{3}{*}{$x=3$} & \multirow{3}{*}{3.394} & $65 \beta\left(\mathrm{O}_{2 \mathrm{p}}\right) \rightarrow 66 \beta(\pi)$ & 99.26 & \multirow{3}{*}{ None } & \multirow{3}{*}{ None } & \multirow{3}{*}{ None } \\
\hline & & $65 \beta\left(\mathrm{O}_{2 \mathrm{p}}\right) \rightarrow 67 \beta\left(\mathrm{Ti}_{3 \mathrm{~d}}\right)$ & 0.51 & & & \\
\hline & & $64 \beta\left(\mathrm{O}_{2 \mathrm{p}}\right) \rightarrow 66 \beta(\pi)$ & 0.04 & & & \\
\hline \multirow{3}{*}{$x=4$} & \multirow{3}{*}{3.484} & $79 \beta\left(\mathrm{O}_{2 \mathrm{p}}\right) \rightarrow 83 \beta\left(\mathrm{Ti}_{3 \mathrm{~d}}\right)$ & 35.61 & \multirow{3}{*}{ None } & \multirow{3}{*}{ None } & \multirow{3}{*}{ None } \\
\hline & & $79 \beta\left(\mathrm{O}_{2 \mathrm{p}}\right) \rightarrow 83 \beta\left(\mathrm{Ti}_{3 \mathrm{~d}}\right)$ & 34.95 & & & \\
\hline & & $71 \beta\left(\mathrm{O}_{2 \mathrm{p}}\right) \rightarrow 80 \beta\left(\mathrm{Ti}_{3 \mathrm{~d}}\right)$ & 7.52 & & & \\
\hline \multirow{3}{*}{$x=5$} & \multirow{3}{*}{3.488} & $94 \alpha[\pi(\mathrm{IS})] \rightarrow 100 \alpha\left(\mathrm{Ti}_{3 \mathrm{~d}}\right)$ & 77.84 & \multirow{3}{*}{2.698} & $94 \alpha[\pi(\mathrm{IS})] \rightarrow 96 \alpha\left(\mathrm{Ti}_{3 \mathrm{~d}}\right)$ & 93.94 \\
\hline & & $94 \alpha[\pi(\mathrm{IS})] \rightarrow 103 \alpha\left(\mathrm{Ti}_{3 \mathrm{~d}}\right)$ & 8.35 & & $94 \alpha[\pi(\mathrm{IS})] \rightarrow 95 \alpha\left(\mathrm{Ti}_{3 \mathrm{~d}}\right)$ & 4.89 \\
\hline & & $94 \alpha[\pi(\mathrm{IS})] \rightarrow 99 \alpha\left(\mathrm{Ti}_{3 \mathrm{~d}}\right)$ & 5.07 & & None & None \\
\hline \multirow{3}{*}{$x=6$} & \multirow{3}{*}{3.486} & $108 \alpha[\pi(\mathrm{IS})] \rightarrow 116 \alpha\left(\mathrm{Ti}_{3 \mathrm{~d}}\right)$ & 57.10 & \multirow{3}{*}{2.749} & $108 \alpha[\pi(\mathrm{IS})] \rightarrow 110 \alpha\left(\mathrm{Ti}_{3 \mathrm{~d}}\right)$ & 90.98 \\
\hline & & $108 \alpha[\pi(\mathrm{IS})] \rightarrow 115 \alpha\left(\mathrm{Ti}_{3 \mathrm{~d}}\right)$ & 32.33 & & $108 \alpha[\pi(\mathrm{IS})] \rightarrow 112 \alpha\left(\mathrm{Ti}_{3 \mathrm{~d}}\right)$ & 3.58 \\
\hline & & $108 \alpha[\pi(\mathrm{IS})] \rightarrow 114 \alpha\left(\mathrm{Ti}_{3 \mathrm{~d}}\right)$ & 4.88 & & $108 \alpha[\pi(\mathrm{IS})] \rightarrow 111 \alpha\left(\mathrm{Ti}_{3 \mathrm{~d}}\right)$ & 3.56 \\
\hline \multirow{3}{*}{$x=7$} & \multirow{3}{*}{3.430} & $122 \alpha[\pi(\mathrm{IS})] \rightarrow 133 \alpha\left(\mathrm{Ti}_{3 \mathrm{~d}}\right)$ & 82.38 & \multirow{3}{*}{2.689} & $122 \alpha[\pi(\mathrm{IS})] \rightarrow 125 \alpha\left(\mathrm{Ti}_{3 \mathrm{~d}}\right)$ & 97.71 \\
\hline & & $122 \alpha[\pi(\mathrm{IS})] \rightarrow 132 \alpha\left(\mathrm{Ti}_{3 \mathrm{~d}}\right)$ & 4.20 & & $122 \alpha[\pi(\mathrm{IS})] \rightarrow 126 \alpha\left(\mathrm{Ti}_{3 \mathrm{~d}}\right)$ & 1.35 \\
\hline & & $122 \alpha[\pi(\mathrm{IS})] \rightarrow 129 \alpha\left(\mathrm{Ti}_{3 \mathrm{~d}}\right)$ & 3.65 & & $122 \alpha[\pi(\mathrm{IS})] \rightarrow 132 \alpha\left(\mathrm{Ti}_{3 \mathrm{~d}}\right)$ & 0.26 \\
\hline \multirow{3}{*}{$x=8$} & \multirow{3}{*}{3.466} & $136 \alpha[\pi(\mathrm{IS})] \rightarrow 148 \alpha\left(\mathrm{Ti}_{3 \mathrm{~d}}\right)$ & 60.32 & \multirow{3}{*}{2.530} & $136 \alpha[\pi(\mathrm{IS})] \rightarrow 138 \alpha\left(\mathrm{Ti}_{3 \mathrm{~d}}\right)$ & 96.97 \\
\hline & & $136 \alpha[\pi(\mathrm{IS})] \rightarrow 147 \alpha\left(\mathrm{Ti}_{3 \mathrm{~d}}\right)$ & 38.41 & & $136 \alpha[\pi(\mathrm{IS})] \rightarrow 137 \alpha\left(\mathrm{Ti}_{3 \mathrm{~d}}\right)$ & 2.28 \\
\hline & & $136 \alpha[\pi(\mathrm{IS})] \rightarrow 154 \alpha\left(\mathrm{Ti}_{3 \mathrm{~d}}\right)$ & 0.28 & & $136 \alpha[\pi(\mathrm{IS})] \rightarrow 140 \alpha\left(\mathrm{Ti}_{3 \mathrm{~d}}\right)$ & 0.25 \\
\hline \multirow{3}{*}{$x=9$} & \multirow{3}{*}{3.442} & $150 \alpha[\pi(\mathrm{IS})] \rightarrow 164 \alpha\left(\mathrm{Ti}_{3 \mathrm{~d}}\right)$ & 72.03 & \multirow{3}{*}{2.718} & $150 \alpha[\pi(\mathrm{IS})] \rightarrow 156 \alpha\left(\mathrm{Ti}_{3 \mathrm{~d}}\right)$ & 78.26 \\
\hline & & $150 \alpha[\pi(\mathrm{IS})] \rightarrow 167 \alpha\left(\mathrm{Ti}_{3 \mathrm{~d}}\right)$ & 10.50 & & $150 \alpha[\pi(\mathrm{IS})] \rightarrow 155 \alpha\left(\mathrm{Ti}_{3 \mathrm{~d}}\right)$ & 5.80 \\
\hline & & $150 \alpha[\pi(\mathrm{IS})] \rightarrow 162 \alpha\left(\mathrm{Ti}_{3 \mathrm{~d}}\right)$ & 4.04 & & $150 \alpha[\pi(\mathrm{IS})] \rightarrow 157 \alpha\left(\mathrm{Ti}_{3 \mathrm{~d}}\right)$ & 3.70 \\
\hline \multirow{3}{*}{$x=10$} & & $164 \alpha[\pi(\mathrm{IS})] \rightarrow 183 \alpha\left(\mathrm{Ti}_{3 \mathrm{~d}}\right)$ & 91.77 & & $164 \alpha[\pi(\mathrm{IS})] \rightarrow 174 \alpha\left(\mathrm{Ti}_{3 \mathrm{~d}}\right)$ & 76.06 \\
\hline & 3.490 & $164 \alpha[\pi(\mathrm{IS})] \rightarrow 184 \alpha\left(\mathrm{Ti}_{3 \mathrm{~d}}\right)$ & 3.76 & 2.722 & $164 \alpha[\pi(\mathrm{IS})] \rightarrow 175 \alpha\left(\mathrm{Ti}_{3 \mathrm{~d}}\right)$ & 12.58 \\
\hline & & $164 \alpha[\pi(\mathrm{IS})] \rightarrow 185 \alpha\left(\mathrm{Ti}_{3 \mathrm{~d}}\right)$ & 1.42 & & $164 \alpha[\pi(\mathrm{IS})] \rightarrow 173 \alpha\left(\mathrm{Ti}_{3 \mathrm{~d}}\right)$ & 8.73 \\
\hline & & $178 \alpha[\pi(\mathrm{IS})] \rightarrow 198 \alpha\left(\mathrm{Ti}_{3 \mathrm{~d}}\right)$ & 32.74 & & $178 \alpha[\pi(\mathrm{IS})] \rightarrow 185 \alpha\left(\mathrm{Ti}_{3 \mathrm{~d}}\right)$ & 76.28 \\
\hline$x=11$ & 3.477 & $178 \alpha[\pi(\mathrm{IS})] \rightarrow 197 \alpha\left(\mathrm{Ti}_{3 \mathrm{~d}}\right)$ & 25.75 & 2.631 & $178 \alpha[\pi(\mathrm{IS})] \rightarrow 184 \alpha\left(\mathrm{Ti}_{3 \mathrm{~d}}\right)$ & 15.26 \\
\hline & & $178 \alpha[\pi(\mathrm{IS})] \rightarrow 199 \alpha\left(\mathrm{Ti}_{3 \mathrm{~d}}\right)$ & 22.14 & & $178 \alpha[\pi(\mathrm{IS})] \rightarrow 183 \alpha\left(\mathrm{Ti}_{3 \mathrm{~d}}\right)$ & 5.72 \\
\hline & & $192 \alpha[\pi(\mathrm{IS})] \rightarrow 211 \alpha\left(\mathrm{Ti}_{3 \mathrm{~d}}\right)$ & 71.93 & & $192 \alpha[\pi(\mathrm{IS})] \rightarrow 201 \alpha\left(\mathrm{Ti}_{3 \mathrm{~d}}\right)$ & 69.73 \\
\hline$x=12$ & 3.440 & $192 \alpha[\pi(\mathrm{IS})] \rightarrow 210 \alpha\left(\mathrm{Ti}_{3 \mathrm{~d}}\right)$ & 6.30 & 2.678 & $192 \alpha[\pi(\mathrm{IS})] \rightarrow 200 \alpha\left(\mathrm{Ti}_{3 \mathrm{~d}}\right)$ & 23.20 \\
\hline & & $192 \alpha[\pi(\mathrm{IS})] \rightarrow 212 \alpha\left(\mathrm{Ti}_{3 \mathrm{~d}}\right)$ & 4.28 & & $192 \alpha[\pi(\mathrm{IS})] \rightarrow 198 \alpha\left(\mathrm{Ti}_{3 \mathrm{~d}}\right)$ & 2.46 \\
\hline & & $206 \alpha[\pi(\mathrm{IS})] \rightarrow 233 \alpha\left(\mathrm{Ti}_{3 \mathrm{~d}}\right)$ & 52.24 & & $206 \alpha[\pi(\mathrm{IS})] \rightarrow 220 \alpha\left(\mathrm{Ti}_{3 \mathrm{~d}}\right)$ & 61.92 \\
\hline$x=13$ & 3.486 & $206 \alpha[\pi(\mathrm{IS})] \rightarrow 231 \alpha\left(\mathrm{Ti}_{3 \mathrm{~d}}\right)$ & 31.93 & 2.716 & $206 \alpha[\pi(\mathrm{IS})] \rightarrow 219 \alpha\left(\mathrm{Ti}_{3 \mathrm{~d}}\right)$ & 19.79 \\
\hline & & $206 \alpha[\pi(\mathrm{IS})] \rightarrow 228 \alpha\left(\mathrm{Ti}_{3 \mathrm{~d}}\right)$ & 6.01 & & $206 \alpha[\pi(\mathrm{IS})] \rightarrow 221 \alpha\left(\mathrm{Ti}_{3 \mathrm{~d}}\right)$ & 7.68 \\
\hline & & $220 \alpha[\pi(\mathrm{IS})] \rightarrow 247 \alpha\left(\mathrm{Ti}_{3 \mathrm{~d}}\right)$ & 73.45 & & $220 \alpha[\pi(\mathrm{IS})] \rightarrow 231 \alpha\left(\mathrm{Ti}_{3 \mathrm{~d}}\right)$ & 56.72 \\
\hline$x=14$ & 3.484 & $220 \alpha[\pi(\mathrm{IS})] \rightarrow 245 \alpha\left(\mathrm{Ti}_{3 \mathrm{~d}}\right)$ & 7.91 & 2.721 & $220 \alpha[\pi(\mathrm{IS})] \rightarrow 233 \alpha\left(\mathrm{Ti}_{3 \mathrm{~d}}\right)$ & 18.27 \\
\hline & & $220 \alpha[\pi(\mathrm{IS})] \rightarrow 251 \alpha\left(\mathrm{Ti}_{3 \mathrm{~d}}\right)$ & 2.28 & & $220 \alpha[\pi(\mathrm{IS})] \rightarrow 232 \alpha\left(\mathrm{Ti}_{3 \mathrm{~d}}\right)$ & 7.73 \\
\hline & & $234 \alpha[\pi(\mathrm{IS})] \rightarrow 263 \alpha\left(\mathrm{Ti}_{3 \mathrm{~d}}\right)$ & 53.89 & & $234 \alpha[\pi(\mathrm{IS})] \rightarrow 247 \alpha\left(\mathrm{Ti}_{3 \mathrm{~d}}\right)$ & 76.75 \\
\hline$x=15$ & 3.431 & $234 \alpha[\pi(\mathrm{IS})] \rightarrow 262 \alpha\left(\mathrm{Ti}_{3 \mathrm{~d}}\right)$ & 17.04 & 2.721 & $234 \alpha[\pi(\mathrm{IS})] \rightarrow 250 \alpha\left(\mathrm{Ti}_{3 \mathrm{~d}}\right)$ & 13.44 \\
\hline & & $234 \alpha[\pi(\mathrm{IS})] \rightarrow 264 \alpha\left(\mathrm{Ti}_{3 \mathrm{~d}}\right)$ & 12.70 & & $234 \alpha[\pi(\mathrm{IS})] \rightarrow 248 \alpha\left(\mathrm{Ti}_{3 \mathrm{~d}}\right)$ & 5.84 \\
\hline
\end{tabular}


Table S4. The transferred negative charges (e) between fragments in $\left(\mathrm{TiO}_{2}\right)_{x} \mathrm{OC}_{5} \mathrm{H}_{8}{ }^{-}$ $(3 \leq x \leq 15)$ clusters based on the interfragment charge transfer (IFCT) analysis. The diagonal terms correspond to amount of interfragment charge redistribution. The excited states with the excitation energy closest to $355 \mathrm{~nm}$ were chosen.

\begin{tabular}{|c|c|c|c|c|}
\hline \multirow{4}{*}{$\left(\mathrm{TiO}_{2}\right)_{3} \mathrm{OC}_{5} \mathrm{H}_{8}^{-}$} & $\begin{array}{lll}\text { Donor } & \text { Acceptor } \\
\end{array}$ & $\mathrm{C}_{5} \mathrm{H}_{8}$ & $\mathbf{O}$ & $\left(\mathrm{TiO}_{2}\right)_{3}$ \\
\hline & $\mathrm{C}_{5} \mathrm{H}_{8}$ & 0.000 & 0.000 & 0.000 \\
\hline & $\mathbf{O}$ & -0.001 & 0.000 & 0.000 \\
\hline & $\left(\mathrm{TiO}_{2}\right)_{3}$ & -0.953 & -0.008 & -0.038 \\
\hline \multirow{4}{*}{$\left(\mathrm{TiO}_{2}\right)_{4} \mathrm{OC}_{5} \mathrm{H}_{8}{ }^{-}$} & Donor $\quad$ Acceptor & $\mathrm{C}_{5} \mathrm{H}_{8}$ & $\mathbf{O}$ & $\left(\mathrm{TiO}_{2}\right)_{4}$ \\
\hline & $\mathrm{C}_{5} \mathrm{H}_{8}$ & 0.000 & 0.000 & -0.001 \\
\hline & $\mathbf{O}$ & 0.000 & 0.000 & -0.005 \\
\hline & $\left(\mathrm{TiO}_{2}\right)_{4}$ & 0.000 & 0.000 & -0.993 \\
\hline \multirow{4}{*}{$\left(\mathrm{TiO}_{2}\right)_{5} \mathrm{OC}_{5} \mathrm{H}_{8}^{-}$} & $\begin{array}{ll} & \text { Acceptor } \\
\text { Donor }\end{array}$ & $\mathrm{C}_{5} \mathrm{H}_{8}$ & $\mathbf{O}$ & $\left(\mathrm{TiO}_{2}\right)_{5}$ \\
\hline & $\mathrm{C}_{5} \mathrm{H}_{8}$ & -0.070 & -0.012 & -0.886 \\
\hline & $\mathbf{O}$ & -0.002 & 0.000 & -0.020 \\
\hline & $\left(\mathrm{TiO}_{2}\right)_{5}$ & -0.001 & 0.000 & -0.008 \\
\hline \multirow{4}{*}{$\left(\mathrm{TiO}_{2}\right)_{6} \mathrm{OC}_{5} \mathrm{H}_{8}^{-}$} & Donor $\quad$ Acceptor & $\mathrm{C}_{5} \mathrm{H}_{8}$ & $\mathbf{O}$ & $\left(\mathrm{TiO}_{2}\right)_{6}$ \\
\hline & $\mathrm{C}_{5} \mathrm{H}_{8}$ & -0.001 & -0.002 & -0.968 \\
\hline & o & 0.000 & 0.000 & -0.018 \\
\hline & $\left(\mathrm{TiO}_{2}\right)_{6}$ & 0.000 & 0.000 & -0.010 \\
\hline \multirow{4}{*}{$\left(\mathrm{TiO}_{2}\right)_{7} \mathrm{OC}_{5} \mathrm{H}_{8}^{-}$} & Donor Acceptor & $\mathrm{C}_{5} \mathrm{H}_{8}$ & $\mathbf{O}$ & $\left(\mathrm{TiO}_{2}\right)_{7}$ \\
\hline & $\mathrm{C}_{5} \mathrm{H}_{8}$ & -0.002 & -0.006 & -0.965 \\
\hline & $\mathbf{O}$ & 0.000 & -0.001 & -0.016 \\
\hline & $\left(\mathrm{TiO}_{2}\right)_{7}$ & 0.000 & 0.000 & 0.009 \\
\hline \multirow{4}{*}{$\left(\mathrm{TiO}_{2}\right)_{8} \mathrm{OC}_{5} \mathrm{H}_{8}^{-}$} & Donor $\quad$ Acceptor & $\mathrm{C}_{5} \mathrm{H}_{8}$ & $\mathbf{O}$ & $\left(\mathrm{TiO}_{2}\right)_{8}$ \\
\hline & $\mathrm{C}_{5} \mathrm{H}_{8}$ & 0.000 & -0.001 & -0.972 \\
\hline & O & 0.000 & 0.000 & -0.016 \\
\hline & $\left(\mathrm{TiO}_{2}\right)_{8}$ & 0.000 & 0.000 & -0.010 \\
\hline \multirow{4}{*}{$\left(\mathrm{TiO}_{2}\right)_{9} \mathrm{OC}_{5} \mathrm{H}_{8}^{-}$} & Donor $\quad$ Acceptor & $\mathrm{C}_{5} \mathrm{H}_{8}$ & $\mathbf{O}$ & $\left(\mathrm{TiO}_{2}\right)_{9}$ \\
\hline & $\mathrm{C}_{5} \mathrm{H}_{8}$ & -0.020 & -0.014 & -0.931 \\
\hline & $\mathbf{O}$ & 0.000 & 0.000 & -0.022 \\
\hline & $\left(\mathrm{TiO}_{2}\right)_{9}$ & 0.000 & 0.000 & -0.012 \\
\hline \multirow{4}{*}{$\left(\mathrm{TiO}_{2}\right)_{10} \mathrm{OC}_{5} \mathrm{H}_{8}^{-}$} & Donor $\quad$ Acceptor & $\mathrm{C}_{5} \mathrm{H}_{8}$ & $\mathbf{O}$ & $\left(\mathrm{TiO}_{2}\right)_{10}$ \\
\hline & $\mathrm{C}_{5} \mathrm{H}_{8}$ & 0.000 & 0.000 & -0.971 \\
\hline & o & 0.000 & 0.000 & -0.018 \\
\hline & $\left(\mathrm{TiO}_{2}\right)_{10}$ & 0.000 & 0.000 & -0.011 \\
\hline \multirow{4}{*}{$\left(\mathrm{TiO}_{2}\right)_{11} \mathrm{OC}_{5} \mathrm{H}_{8}^{-}$} & Donor Acceptor & $\mathrm{C}_{5} \mathrm{H}_{8}$ & $\mathbf{O}$ & $\left(\mathrm{TiO}_{2}\right)_{11}$ \\
\hline & $\mathrm{C}_{5} \mathrm{H}_{8}$ & -0.062 & -0.020 & -0.886 \\
\hline & $\mathbf{O}$ & -0.001 & 0.000 & -0.020 \\
\hline & $\left(\mathrm{TiO}_{2}\right)_{11}$ & -0.001 & 0.000 & -0.009 \\
\hline \multirow{2}{*}{$\left(\mathrm{TiO}_{2}\right)_{12} \mathrm{OC}_{5} \mathrm{H}_{8}^{-}$} & Donor $\quad$ Acceptor & $\mathrm{C}_{5} \mathrm{H}_{8}$ & O & $\left(\mathrm{TiO}_{2}\right)_{12}$ \\
\hline & $\mathrm{C}_{5} \mathrm{H}_{8}$ & -0.068 & -0.010 & -0.887 \\
\hline
\end{tabular}




\begin{tabular}{|c|c|c|c|c|}
\hline & $\begin{array}{c}\mathbf{O} \\
\left(\mathrm{TiO}_{2}\right)_{12} \\
\end{array}$ & $\begin{array}{l}-0.001 \\
-0.001 \\
\end{array}$ & $\begin{array}{l}0.000 \\
0.000 \\
\end{array}$ & $\begin{array}{l}-0.018 \\
-0.014 \\
\end{array}$ \\
\hline \multirow{4}{*}{$\left(\mathrm{TiO}_{2}\right)_{13} \mathrm{OC}_{5} \mathrm{H}_{8}^{-}$} & $\begin{array}{lll}\text { Donor } & \text { Acceptor } \\
\end{array}$ & $\mathrm{C}_{5} \mathrm{H}_{8}$ & $\mathbf{O}$ & $\left(\mathrm{TiO}_{2}\right)_{13}$ \\
\hline & $\mathrm{C}_{5} \mathrm{H}_{8}$ & -0.003 & -0.004 & -0.963 \\
\hline & $\mathbf{O}$ & 0.000 & 0.000 & -0.017 \\
\hline & $\left(\mathrm{TiO}_{2}\right)_{13}$ & 0.000 & 0.000 & -0.013 \\
\hline \multirow{4}{*}{$\left(\mathrm{TiO}_{2}\right)_{14} \mathrm{OC}_{5} \mathrm{H}_{8}^{-}$} & Donor $\quad$ Acceptor & $\mathrm{C}_{5} \mathrm{H}_{8}$ & $\mathbf{O}$ & $\left(\mathrm{TiO}_{2}\right)_{14}$ \\
\hline & $\mathrm{C}_{5} \mathrm{H}_{8}$ & -0.006 & -0.012 & -0.952 \\
\hline & $\mathbf{O}$ & 0.000 & 0.000 & -0.019 \\
\hline & $\left(\mathrm{TiO}_{2}\right)_{14}$ & 0.000 & 0.000 & -0.011 \\
\hline \multirow{4}{*}{$\left(\mathrm{TiO}_{2}\right)_{15} \mathrm{OC}_{5} \mathrm{H}_{8}{ }^{-}$} & Donor $\quad$ Acceptor & $\mathrm{C}_{5} \mathrm{H}_{8}$ & $\mathbf{O}$ & $\left(\mathrm{TiO}_{2}\right)_{15}$ \\
\hline & $\mathrm{C}_{5} \mathrm{H}_{8}$ & -0.009 & -0.002 & -0.956 \\
\hline & $\mathbf{O}$ & 0.000 & 0.000 & -0.021 \\
\hline & $\left(\mathrm{TiO}_{2}\right)_{15}$ & 0.000 & 0.000 & -0.011 \\
\hline
\end{tabular}




\section{Reference.}

(1) Ma, J.-B.; Xu, B.; Meng, J.-H.; Wu, X.-N.; Ding, X.-L.; Li, X.-N.; He, S.-G., Reactivity of Atomic Oxygen Radical Anions Bound to Titania and Zirconia Nanoparticles in the Gas Phase: Low-Temperature Oxidation of Carbon Monoxide. $J$. Am. Chem. Soc. 2013, 135, 2991-2998.

(2) Lu, T.; Chen, F., Multiwfn: A Multifunctional Wavefunction Analyzer. J. Comput. Chem. 2012, 33, 580-592. 